\title{
Phonon Drag on Dislocations at High Pressures
}

W.G. Wolfer

October 19, 1999

Lawrence

Livermore

National

Laboratory

Approved for public release; further dissemination unlimited 


\section{DISCLAIMER \\ .}

This document was prepared as an account of work sponsored by an agency of the United States Government. Neither the United States Government nor the University of California nor any of their employees, makes any warranty, express or implied, or assumes any legal liability or responsibility for the accuracy, completeness, or usefulness of any information, apparatus, product, or process disclosed, or represents that its use would not infringe privately owned rights. Reference herein to any specific commercial product, process, or service by trade name, trademark, manufacturer, or otherwise, does not necessarily constitute or imply its endorsement, recommendation, or favoring by the United States Government or the University of California. The views and opinions of authors expressed herein do not necessarily state or reflect those of the United States Government or the University of California, and shall not be used for advertising or product endorsement purposes.

Work performed under the auspices of the U. S. Department of Energy by the University of California Lawrence Livermore National Laboratory under Contract W-7405-Eng-48.

This report has been reproduced

directly from the best available copy.

Available to DOE and DOE contractors from the

Office of Scientific and Technical Information

P.O. Box 62, Oak Ridge, TN 37831

Prices available from (423) 576-8401

http://apollo.osti.gov/bridge/

Available to the public from the

National Technical Information Service

U.S. Department of Commerce

5285 Port Royal Rd.,

Springfield, VA 22161

http://www.ntis.gov/

OR

Lawrence Livermore National Laboratory

Technical Information Department's Digital Library

http://www.llnl.gov/tid/Library.html 


\title{
Phonon Drag on Dislocations at High Pressures
}

\author{
W. G. Wolfer \\ Chemistry and Materials Science \\ Lawrence Livermore National Laboratory \\ Livermore, CA 94550. USA
}

Phonon drag on dislocations is the dominant process which determines the flow stress of metals at elevated temperatures and at very high plastic deformation rates. The dependence of the phonon drag on pressure or density is derived using a Mie-Grueneisen equation of state. The phonon drag is shown to increase nearly linearly with temperature but to decrease with density or pressure. Numerical results are presented for its variation for shock-loaded copper and aluminum. In these cases, density and temperature increase simultaneously, resulting in a more modest net increase in the dislocation drag coefficient. Nevertheless, phonon drag increases by more than an order of magnitude during shock deformations which approach melting.

Since the dependencies of elastic modulii and of the phonon drag coefficient on pressure and temperature are fundamentally different, the effect of pressure on the constitutive law for plastic deformation can not simply be accounted for by its effect on the elastic shear modulus. 


\section{Introduction}

The plastic deformation of crystalline materials is caused by the simple slip motion of dislocations. The flow stress required to set dislocations in motion depends on many factors and interactions with numerous crystal defects. In spite of this complexity, it is nevertheless possible to identify the dominant processes of glide mobilization for a given crystal structure type, a given temperature, and a given strain rate. This classification of the dominant deformation mechanisms is now well known and referred to as the Ashby deformation maps ${ }^{1}$. According to these maps, dislocations spend most of their time pinned or trapped by glide obstacles when the strain rate is below $10^{4}$, and very little time in motion. On the other hand, when the strain rate is $10^{5}$ or greater, the free flight time between consecutive arrests becomes of the same order as the waiting time. In this case, the flow stress becomes dependent on the drag exerted by the lattice vibrations and, at cryogenic temperatures, also by the conduction electrons. These drag forces on the moving dislocations have the same physical origin as do the residual thermal and electrical resistivities of stationary dislocations. In both cases, the phonons or electrons flow past the dislocations which scatter them and thereby dissipate energy.

Plastic deformation at very high strain rates is often achieved under shock loading conditions which also generate very high hydrostatic pressures and temperatures. It is therefore important to understand the effect of high pressure on the phonon drag coefficient. In the present paper, this dependence will be determined assuming a MieGrueneisen equation of state.

The paper is organized as follows. In section 2, a simple constitutive model is described for plastic deformation which incorporates the two major mechanisms in dislocation mobilization, namely thermally activated escape from obstacles or junctions, and overcoming the phonon drag. This fundamental model for dislocation mobility is the basis for many empirical constitutive laws used in plasticity theory. Next, we review in section 3 the theory and the experimental measurements of the phonon drag coefficient, and then derive in section 4 the relationships which determine its pressure and temperature dependence. Numerical results for copper and aluminum are presented in section 5 .

\section{Constitutive Laws for Plastic Deformation}

Dislocation reactions and processes during plastic deformation are extremely complex, and the diversity of phenomena is perhaps one of the richest to be found in materials science. Yet the stress-strain curves of metals exhibit few and simple characteristics which can be described by simple mathematical relationships. Apparently, much of the complexity of the many-defect system becomes statistically averaged at the continuum level, leading to simple, empirical relationships for the plastic constitutive laws. But since there does not yet exist a rigorous statistical theory of an evolving dislocation network, constitutive laws for plastic deformation are still formulated in terms of a single dislocation segment subject to an effective stress. This effective stress is a sum of the externally applied stress and mean-field internal stresses generated by local glide 
obstacles, by other dislocation segments and junctions with them, and by the polarisation stresses of surrounding precipitates, subgrains and grains. In short, the internal stresses have contributions from all length scales except those captured in the applied stress term, and they represent the effect of all the many-defect interactions between one generic dislocation segment with all other defects. The dividing length scale for external and internal stresses is to some extent a matter of choice, as it depends on the resolution desired or appropriate for the continuum stress analysis.

According to Gillis, Gilman, and Taylor ${ }^{2}$, a dislocation segment moves on the average a distance $L$ between consecutive stops at glide obstacles. Denoting the average waiting time as $t_{w}$, and the mean free-flight time between stops by $t_{f}$, the average dislocation velocity is

$$
<v>=L /\left(t_{w}+t_{f}\right)
$$

while the average flight velocity is

$$
\mathrm{v}_{\mathrm{f}}=\mathrm{L} / \mathrm{t}_{\mathrm{f}} .
$$

This flight velocity is determined by the mechanical force $b \tau$ acting on the dislocation and by the drag force $\mathrm{Bv}_{\mathrm{f}}$. Neglecting the very short period of acceleration,

$$
\mathrm{b} \tau=\mathrm{Bv}_{\mathrm{f}} .
$$

Here, $b$ is the Burgers vector, $\tau$ is the resolved shear stress on the glide plane, and B is the drag coefficient.

To derive a constitutive law we follow the arguments of Kroener and Teodosiu ${ }^{3}$. If there is a density of $\rho_{\mathrm{m}}$ dislocation segments which can potentially be mobilized, and if $\rho_{\mathrm{f}}$ is the dislocation density in flight and $\rho_{\omega}=\rho_{\mathrm{m}}-\rho_{\mathrm{f}}$ the density at rest, then

$$
\frac{d \rho_{f}}{d t}=\frac{\rho_{w}}{t_{w}}-\frac{\rho_{f}}{t_{f}}
$$

For time scales longer than $t_{f}$, the solution to eq. (4) is very close to the steady-state solution

$$
\rho_{f}=\frac{t_{f}}{t_{w}} \rho_{w}=\frac{t_{f}}{t_{w}+t_{f}} \rho_{m}
$$

The rate of plastic shear is now given by

$$
\dot{\gamma}=b \rho_{f} v_{f}=b \frac{t_{f}}{t_{w}+t_{f}} \rho_{m} \frac{L}{t_{f}}=\frac{b L \rho_{m}}{t_{w}+t_{f}}
$$

The rate of release of a dislocation segment from its present glide obstacle is assumed to be controlled by a stress-assisted, thermally activated process expressed by the Arrhenius formula 


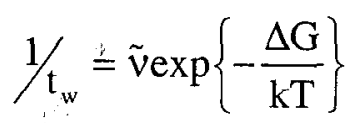

where the attempt frequency of the dislocation segment is given by

$$
\tilde{v}=\frac{b v_{p}}{\lambda}
$$

Here, $v_{D}$ is the Debye frequency and $\lambda$ is a characteristic length parameter of the glide obstacle, for example, the average distance between glide obstacles in the case of fcc metals or the width of a double kink in the case of a screw dislocation in a bcc metal. From eqs.(1) and (2) one obtains

$$
t_{f}=\frac{L B}{b \tau}
$$

If one now inserts eqs. (7) and (9) into eq. (6), one arrives at the constitutive law

$$
\dot{\gamma}=\frac{\mathrm{b}^{2} \rho_{\mathrm{m}}}{\left(\lambda / \mathrm{L} v_{\mathrm{D}}\right) \exp \{\Delta \mathrm{G}(\tau) / \mathrm{kT}\}+(\mathrm{B} / \tau)}
$$

As mentioned above, the shear stress $\tau$ is an effective local stress and written as

$$
\tau=\tau_{\text {Applied }}-\tau_{\mathrm{I}}
$$

The first term is the shear stress due to the applied loads and the one experimentally measured or controlled, and the second term is due to internal sources. This stress is known by various names, e.g. back stress, threshold stress, athermal part of the yield strength, friction stress, etc. Each of these names invokes a somewhat different aspect of the mean-field internal stress and its contributions from many length scales. The reason why the effective stress is written as a difference is the intuitive vicw that plastic flow produced by an applied stress can occur only when it exceeds a given value determined by the state of the internal dislocation microstructure. The constitutive law embodied in eqs. (10) and (11) contain both physical and microstructure parameters as listed in Table 1. The distinction between the two types is not entirely perfect as two parameters fit both categories. The physical parameters have well defined dependencies on pressure and temperature while the microstructure parameters are average quantities with ill defined statistical aspects and dependencies on prior deformation and materials processing variables.

The contributions to the internal stress $\tau_{\mathrm{I}}$ which depend on dislocation-dislocation or defect-dislocation interactions usually scale with the elastic shear modulus. This scaling is also noted when one compares the Ashby deformation maps for materials belonging to the same crystal structure group. When these maps are depicted in reduced units of stress divided by shear modulus and temperature divided by the melting point, the maps become nearly identical. As a result, it is generally assumed that shear stresses used in 
formulating constitutive laws should really be replaced by corresponding elastic shear strains. In other words, eq. (10) should be written as

$$
\dot{\gamma}=\frac{b^{2} \rho_{m}}{\left(\lambda / L v_{D}\right) \exp \{\Delta G(\tau / \mu) / k T\}+(B / \mu)(\mu / \tau)},
$$

where $\mu$ is an appropriate shear modulus. In some constitutive plastic deformation laws based on eq. (12), for example one proposed by Steinberg and Lund ${ }^{4}$, the implicit assumption is made that $B / \mu$ is independent of pressure and temperature. However, as will be shown below, this is not a valid assumption.

Table 1. Physical and Microstructure Parameters

Physical parameters which depend on $\mathrm{P}$ and $\mathrm{T}$
Microstructure parameters which depend on deformation history

$\begin{array}{ll}\mu & \\ \mathrm{b} & \rho_{\mathrm{m}} \text { and } \rho_{\mathrm{f}} \\ \mathrm{B} & \mathrm{L} \\ v_{\mathrm{D}} & \tau_{\mathrm{I}} \\ \lambda & \lambda \\ \Delta \mathrm{G} & \Delta \mathrm{G}\end{array}$

\section{Phonon Drag on Dislocations}

The mechanisms and theoretical approaches to dislocation drag have been reviewed by Nabarro ${ }^{5}$. With regard to the phonon drag, there are two complementary approaches. Mason's approach considers a dislocation moving through a viscous medium. The viscosity is a sum of two contributions: one from the electrons, and one from the phonons. The electron viscosity is determined from theoretical models, and the phonon viscosity is derived from the experimentally measured damping coefficient of sound waves by subtracting the electronic contribution. In this manner, a total dislocation drag coefficient is predicted as a function of temperature. It exhibits a marked increase at cryogenic temperatures where the electronic contributions dominates. However, this increase is in conflict with the direct determination of dislocation damping under a dynamic bias stress, as measured by Hikata et al. ${ }^{6}$. It also is in conflict with the theoretical derivation of the electronic drag coefficient by Kravchenko ${ }^{7}$ and by Brailsford $^{8}$ which gives a temperature-independent value. Hikata et al. have in fact shown that their experimental results are in agreement with this temperature-independent value at cryogenic temperatures, and with a temperature-dependent phonon drag coefficient at high temperature as originally derived by Leibfried ${ }^{9}$ and later on refined by Brailsford ${ }^{10}$. 
In the approach pioneered by Leibfried, the scattering of an elastic wave or phonon by a dislocation is computed. Taking the difference of the scattering for two waves, one propagating in the direction and the other opposite to the moving dislocation, and then averaging over all wave directions and phonon frequencies, he obtained the following phonon drag coefficient:

$$
\mathrm{B}_{\mathrm{ph}}=\frac{\mathrm{q}}{10 \mathrm{c}_{\mathrm{t}}} \bar{\varepsilon}_{\mathrm{ph}}
$$

Here, $q$ is the cross-section of the dislocation core per unit length, $c_{t}$ is the transverse sound speed, and $\bar{\varepsilon}_{\mathrm{ph}}$ is the average thermal phonon energy per unit volume. Qualitative arguments led Leibfried to the conclusion that $\mathrm{q}$ is of the order of the lattice parameter. Brailsford ${ }^{10}$ has provided a much more detailed derivation of phonon drag and shown in the process that Mason's theory is inadequate as it takes not into account that the phonon viscosity is both frequency and wave-vector dependent. In addition, Brailsford's treatment encompasses all the different mechanisms mentioned by Nabarro, and it shows that the phonon scattering mechanism first considered by Leibfried is indeed the dominant one.

Hikata et al. have demonstrated that the Leibfried formula together with a temperature independent electronic drag contribution provide an excellent expression for fitting the dislocation drag measurements in aluminum. We will discuss this comparison of theory and experimental data in section 6.

\section{Temperature and Volume Dependence of the Phonon Drag}

Within the Debye approximation, the average thermal phonon energy can be written as

$$
\bar{\varepsilon}_{\mathrm{ph}}=\frac{3 \hbar}{2 \pi^{2} \overline{\mathrm{c}}^{3}} \int_{0}^{\omega_{\mathrm{D}}} \mathrm{d} \omega \frac{\omega^{3}}{\mathrm{e}^{\hbar \omega / \mathrm{kT}}-1}
$$

Here, $\overline{\mathrm{c}}$ is the average sound speed which can be related to the Debye frequency $\omega_{\mathrm{D}}$ by

$$
\omega_{D}=2 \pi \bar{c}\left(\frac{3 N}{4 \pi V}\right)^{1 / 3}
$$

where $\mathrm{N}$ is the number of atoms in the volume $\mathrm{V}$. It is convenient to introduce now the dimensionless variables

$$
\mathrm{x}=\hbar \omega / \mathrm{kT} \quad \text { and } \quad \mathrm{x}_{\mathrm{D}}=\hbar \omega_{\mathrm{D}} / \mathrm{kT}=\Theta_{\mathrm{D}} / \mathrm{T}
$$

The average thermal phonon energy can then be written as

$$
\bar{\varepsilon}_{\mathrm{ph}}=9 \mathrm{kT}(\mathrm{N} / \mathrm{V}) \mathrm{D}_{3}\left(\mathrm{x}_{\mathrm{D}}\right)
$$

where $D_{3}$ is the Debye integral 


$$
D_{3}\left(x_{D}\right)=\frac{3}{x_{D}^{3}} \int_{0}^{x_{D}} \frac{x^{3} d x}{e^{x}-1}
$$

The Debye temperature $\Theta_{D}$ is a function of the volume, and its dependence can be evaluated from the Grueneisen constant $\Gamma(V)$ according to the equation

$$
\ln \left[\Theta_{D}(V) / \Theta_{D}\left(V_{0}\right)\right]=\int_{V}^{V_{o}} \frac{\Gamma(V)}{V} d V
$$

To complete the volume dependence of the drag coefficient, we use the following approximation for the transverse sound speed in an elastically isotropic material:

$$
\frac{3}{\bar{c}^{3}}=\frac{2}{c_{t}^{3}}+\frac{1}{c_{\ell}^{3}} \cong\left[2+\left(\frac{1-2 v}{2-2 v}\right)^{3 / 2}\right] \frac{1}{c_{t}^{3}} \cong \frac{9}{8 c_{t}^{3}}
$$

Here, $v$ is the Poisson's ratio taken to be equal to $1 / 3$.

Finally, the scattering width of the dislocation changes with the volume according to

$$
q=q_{0}\left(V / V_{0}\right)^{1 / 3}
$$

Using the above formulae we can express the phonon drag coefficient in the following form:

$$
\mathrm{B}_{\mathrm{ph}}=0.209 \mathrm{hq}_{\mathrm{o}} \Omega_{\mathrm{o}}^{-4 / 3} \frac{\mathrm{V}_{\mathrm{o}}}{\mathrm{V}} \mathrm{D}_{\mathrm{E}}\left(\mathrm{x}_{\mathrm{D}}\right)
$$

Here, $\mathrm{h}$ is the Planck's constant, $\Omega_{\mathrm{o}}$ is the atomic volume at standard pressure, and

$$
\mathrm{D}_{\mathrm{E}}\left(\mathrm{x}_{\mathrm{D}}\right)=\mathrm{D}_{3}\left(\mathrm{x}_{\mathrm{D}}\right) / \mathrm{x}_{\mathrm{D}}
$$

Let us denote the phonon drag coefficient at standard pressure and temperature $(273 \mathrm{~K})$ by $\mathrm{B}_{\mathrm{ph}}^{\mathrm{o}}$. Then the volume or pressure dependence can be found from the simple relationship

$$
B_{p h}(V, T)=B_{p h}^{\circ} \frac{V_{o}}{V} \frac{D_{E}\left(x_{D}\right)}{D_{E}\left(x_{D}^{\circ}\right)}
$$

This relationship is evaluated in the following section.

\section{Phonon Drag Coefficient during Shock-Loading}

Phonon drag on dislocations determines the flow stress only at high deformation rates. Rates on the order of $10^{5}$ or higher are typically achieved under shock-loading conditions which also result in high hydrostatic pressures and high temperatures. The variation of 
the phonon drag coefficient under shock-loading depends then on the simultaneous change of density and temperature, i.e. on the Hugoniot and the equation of state. In the following numerical evaluation, we use the equation of state data for copper and aluminum as compiled by Eliezer et al. ${ }^{11}$.

Figure 1 shows the Hugoniot curve for $\mathrm{Cu}$ and Figure 2 the associated temperature, the Debye temperature, and the melt temperature. The latter is obtained from the theoretical work of Moriarty ${ }^{12}$. Melting is predicted to occur at a pressure of about $2.3 \mathrm{Mb}$. The variation of the phonon drag coefficient over the pressure range of solid copper is shown in Figure 3. It is seen that the drag coefficient increases with the pressure up to a factor of about 14.

The corresponding results for aluminum are displayed in Figure 4 for the Hugoniot. The temperature along the Hugoniot, the Debye temperature as well as two melt temperatures have been predicted by Moriarty, Young, and Ross ${ }^{13}$, and they are shown in Figure 5. The curve denoted by GPT is obtained with the generalized pseudopotential theory of Moriarty ${ }^{14}$, while the curve labeled HLP is a prediction using the Harrison local pseudopotential. Note that the melt temperature predictions were not adjusted to the known melting point of aluminum, and they are somewhat higher at standard density. Melting on shock compression is predicted to occur at a compression ratio of 1.74 or a temperature of about $5500 \mathrm{~K}$. Figure 6 shows the variation of the phonon drag coefficient. It is seen that the drag coefficient for $\mathrm{Al}$ increases by a factor of 25 before melting occurs.

\section{Discussions}

The phonon drag coefficient according to eq. (24) is both a strong function of temperature and density or pressure. To display these dependencies separately, let us consider the case for copper. Figure 7 shows the temperature dependence for a constant density, neglecting the small density changes due to thermal expansion. It is seen that the phonon drag coefficient increases linearly with temperatures for values of the order of the Debye temperature or greater. It changes roughly by an factor of 6 for a $1000 \mathrm{~K}$ tempcrature increase. The reason for the increase is that the phonon density rises with temperature.

In contrast, when the density of the material increases at constant temperature, the phonon drag coefficient declines. Over the density range where copper remains in the solid state during a typical shock deformation, the coefficient drops roughly by an order of magnitude, as can be seen from Figure 8. The reason for the drop in phonon drag with density is the increase in the Debye temperature, see Figure 2, or the reduction in the phonon density.

In shock deformation experiments where both the temperature and the density increase simultaneously, there is a partial cancellation of the two effects on the phonon drag, and it is for this reason that the net change is, for example, only a factor of 14 in the case of copper.

One of the major objectives of this investigation was to determine the appropriate dependence of the flow stress with pressure or density. As pointed out in section 2 and in the formulation of the constitutive law of eq. (24), it is often assumed that the pressure dependence is solely contained in the shear modulus because plastic deformation depends on $(\tau / \mu)$. Implicit in this assumption is then that other materials parameters entering the 
constitutive law are independent of pressure or density. We have seen that these assumptions do not apply to the phonon drag term. In order to obtain the correct pressure dependence, one must examine the physical basis for the phonon drag on a dislocation and derive or determine its true pressure and temperature dependencies.

This raises the question as to the pressure dependence of the thermally activated process in the constitutive deformation law, i. e. when the phonon drag is absent as in the Steinberg-Guinan model ${ }^{15}$. The present assumption that this dependence can be accounted for with a pressure-dependent shear modulus needs to be critically examined. We finally notice, as did Hikata et al. earlier, that the Leibfried equation (13) provides excellent quantitative predictions for the phonon drag coefficient. Figure 9 shows the experimental results for aluminum of Hikata et al. Using a scattering cross section of $\mathrm{q}$ equal to one Burgers vector and adding to equation (13) a constant electron drag coefficient of 1.4 dynes sec/ $\mathrm{cm}^{2}$, the solid curve in Figure 9 is obtained. The agreement between the theoretical and experimental results is obvious.

\section{Acknowledgements}

The author thanks Drs. J. A. Moriarty and J. Stölken for critical reading of the paper and valuable suggestions for its improvement.

This work was performed under the auspices of the U.S. Department of Energy and the Lawrence Livermore National Laboratory under contract number W-7405-ENG-48.

\section{References}

1) H. J. Frost and M. F. Ashby, Deformation-Mechanism Maps, Pergamon Press, Oxford, 1982

2) P.P. Gillis, J.J. Gilman, and J. W. Taylor, Phil Mag. 20 (1969) 279

3) E. Kroener and C. Teodosiu, "Lattice defect approach to plasticity and viscoplasticity", in Problems of Plasticity, cd. by A. Sawczuk, Noordhoff Internat. Publ., Leyden, 1974, p. 45

4) D.J. Steinberg and C.M. Lund, J. Appl. Phys. 65 (1989) 1528

5) F.R.N. Nabarro, Theory of Crystal Dislocations, Oxford University Press, Oxford, 1967 , chapter 7

6) A. Hikata, R.A. Johnson, and C. Elbaum, Phys. Rev. B 2 (1970) 4856

7) V.Ya. Kravchenko, Sov. Phys. Solid State 8 (1966) 740

8) A.D. Brailsford, Phys. Rev. 186 (1969) 959

9) G. Leibfried, Z. Physik 127 (1950) 344

10) A.D. Brailsford, J. Appl. Phys. 41 (1970) 4439; J. Appl. Phys. 43 (1972) 1380

11) S. Eliezer, A. Ghatak, and H. Hora; "An Introduction to Equations of State: Theory and Applications", Cambridge Univ. Press, Cambridge 1986, Chapter 13.

12) J.A. Moriarty; in "Shock Waves in Condensed Matter", ed. by Y.M. Gupta, Plenum Publishing Corp., 1986, p. 101

13) J.A. Moriarty, D.A. Young, and M. Ross, Phys. Rev. B 30 (1984) 578

14) J.A. Moriarty, Phys. Rev. B16 (1977) 2537, B 26 (1982)1754, B 38 (1984) 3199

15) D.J. Steinberg, S.G. Cochran, and M.W. Guinan, J. Appl. Phys. 51 (1980) 1498 


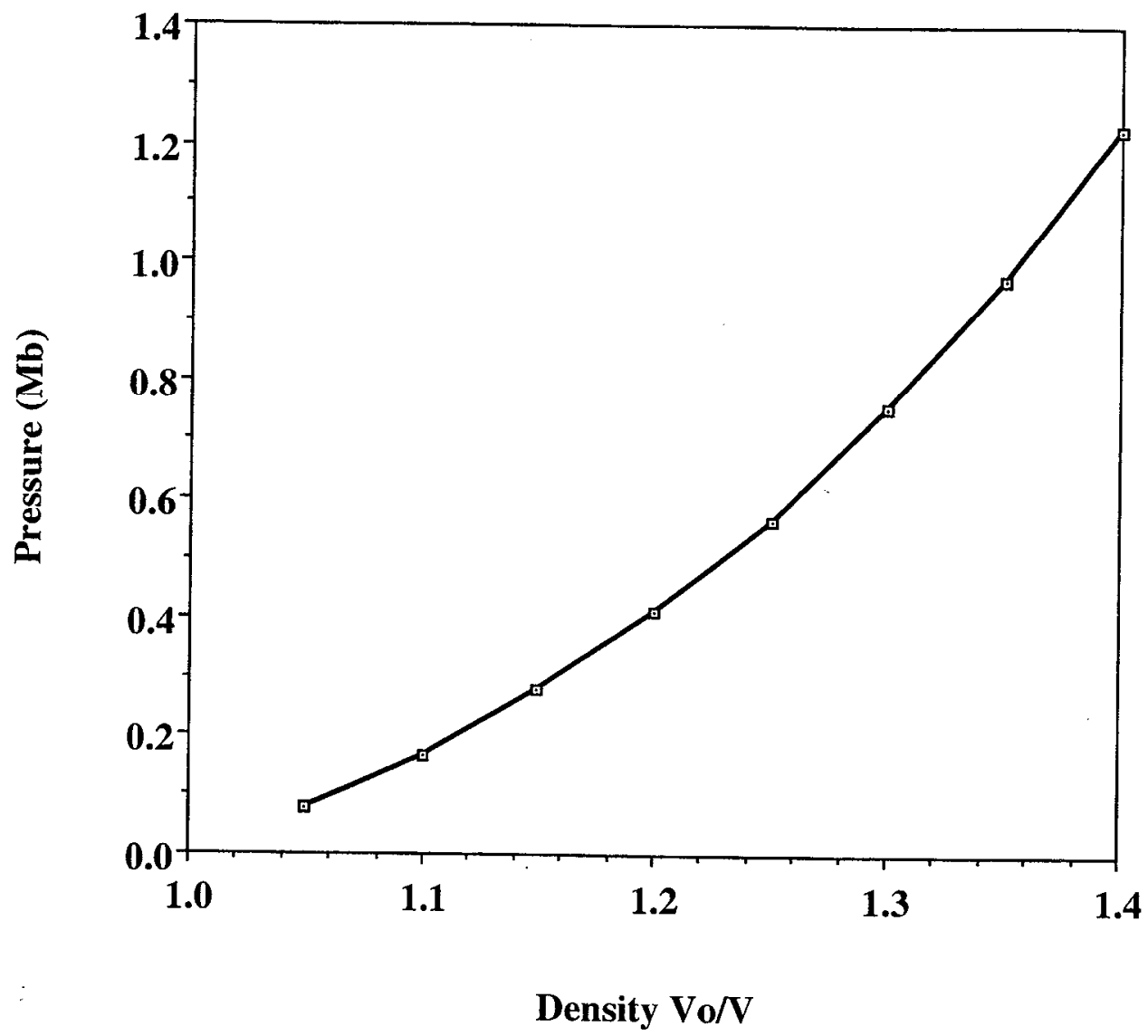

Figure 1. Hugoniot for $\mathrm{Cu}$ 


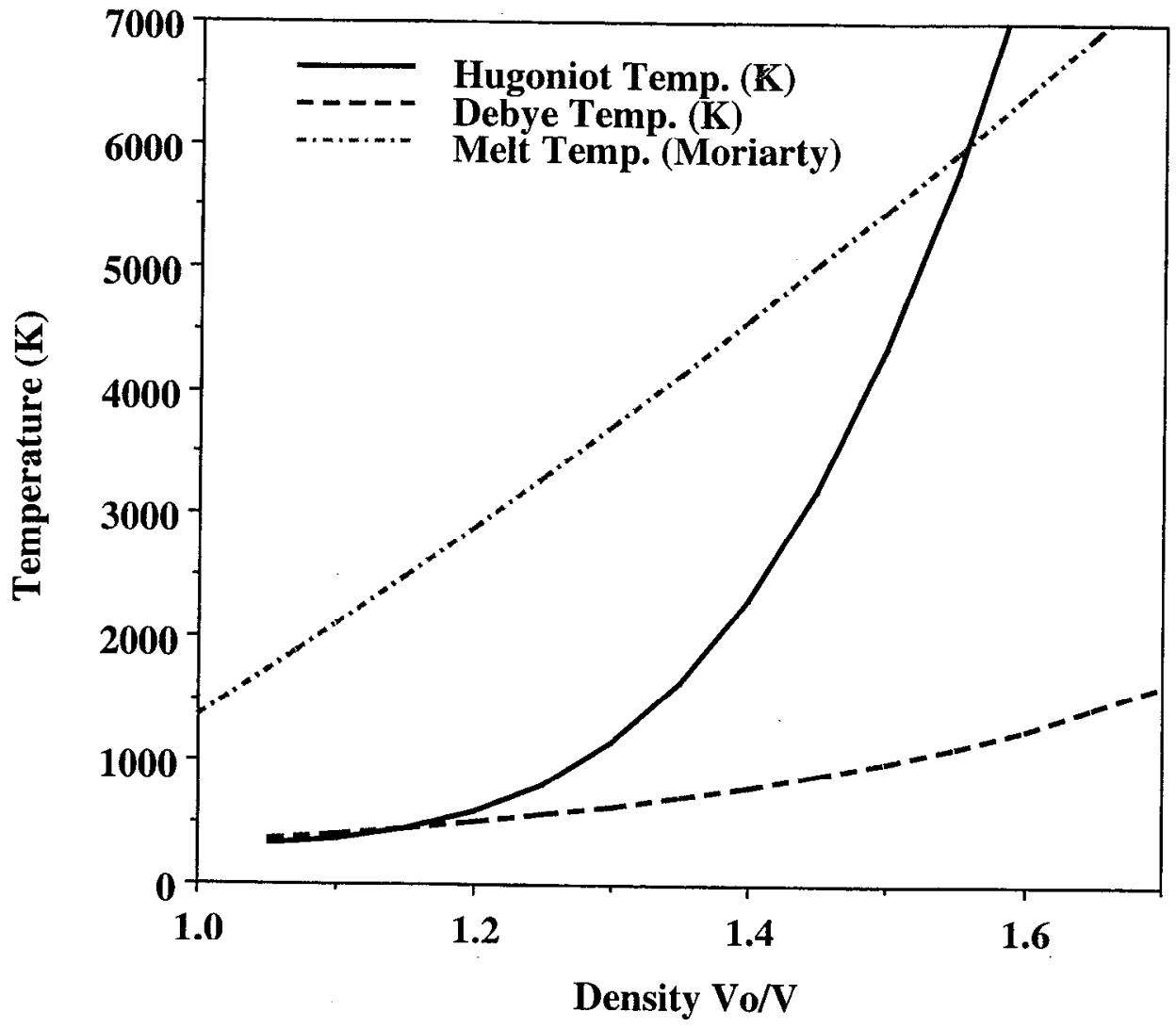

Figure 2. The Hugoniot, Debye, and Melt temperatures for $\mathrm{Cu}$. 


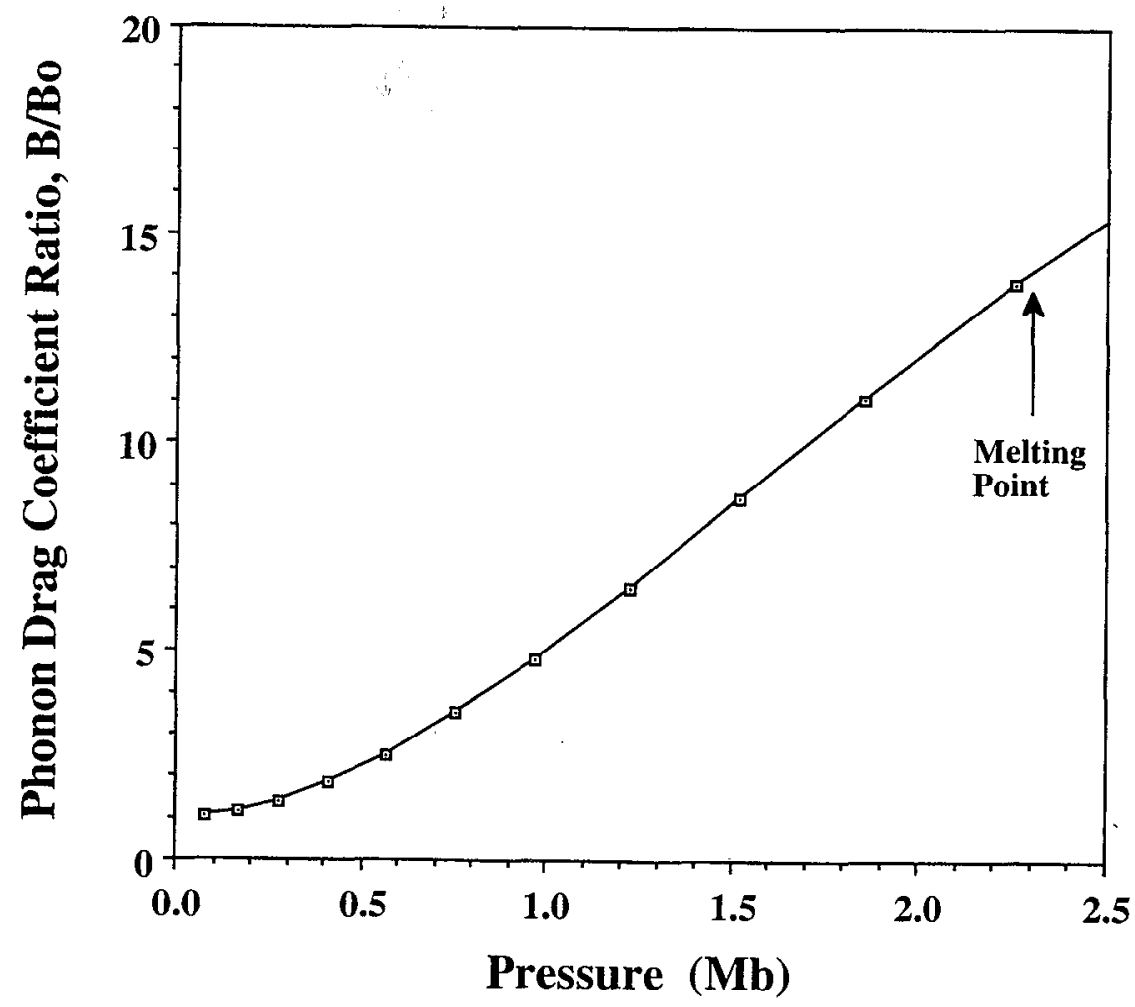

Figure 3. Variation of the Drag Coefficient with Pressure for Shock-Loaded $\mathrm{Cu}$. The Debye temperature at standard conditions is $329 \mathrm{~K}$. 


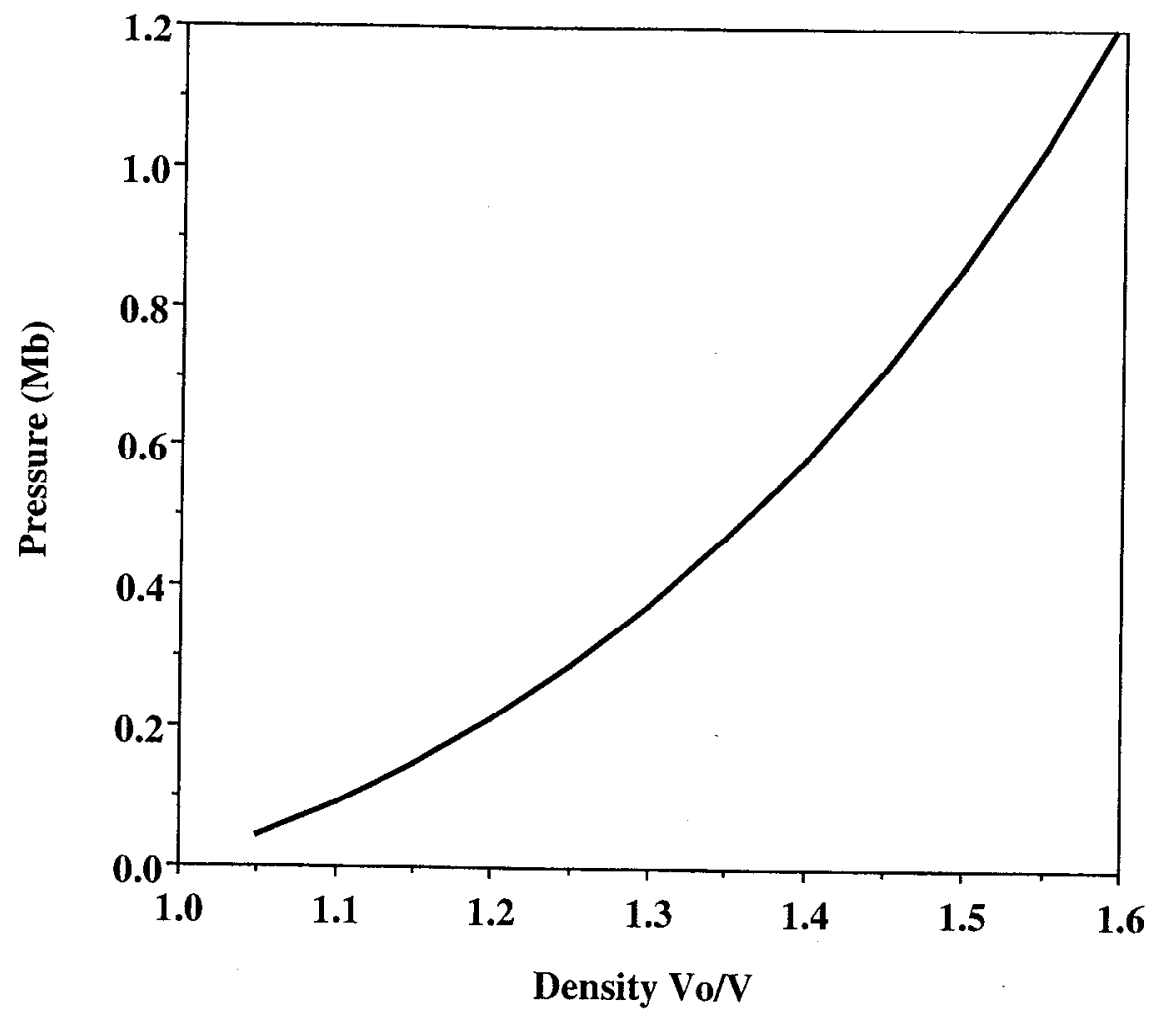

Figure 4. Hugoniot for $\mathrm{Al}$ 


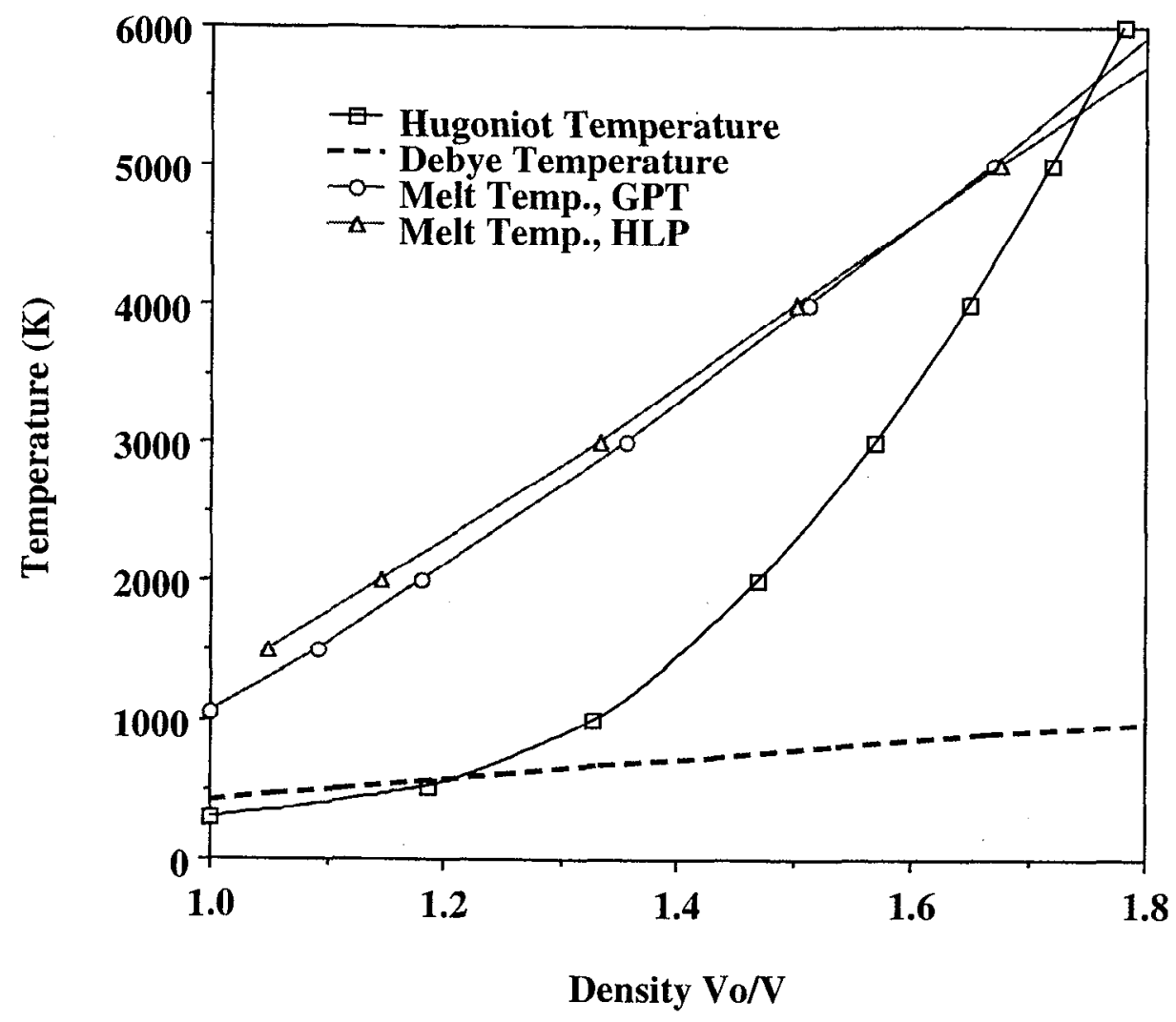

Figure 5. The Hugoniot, Debye, and Melt Temperatures for Al. 


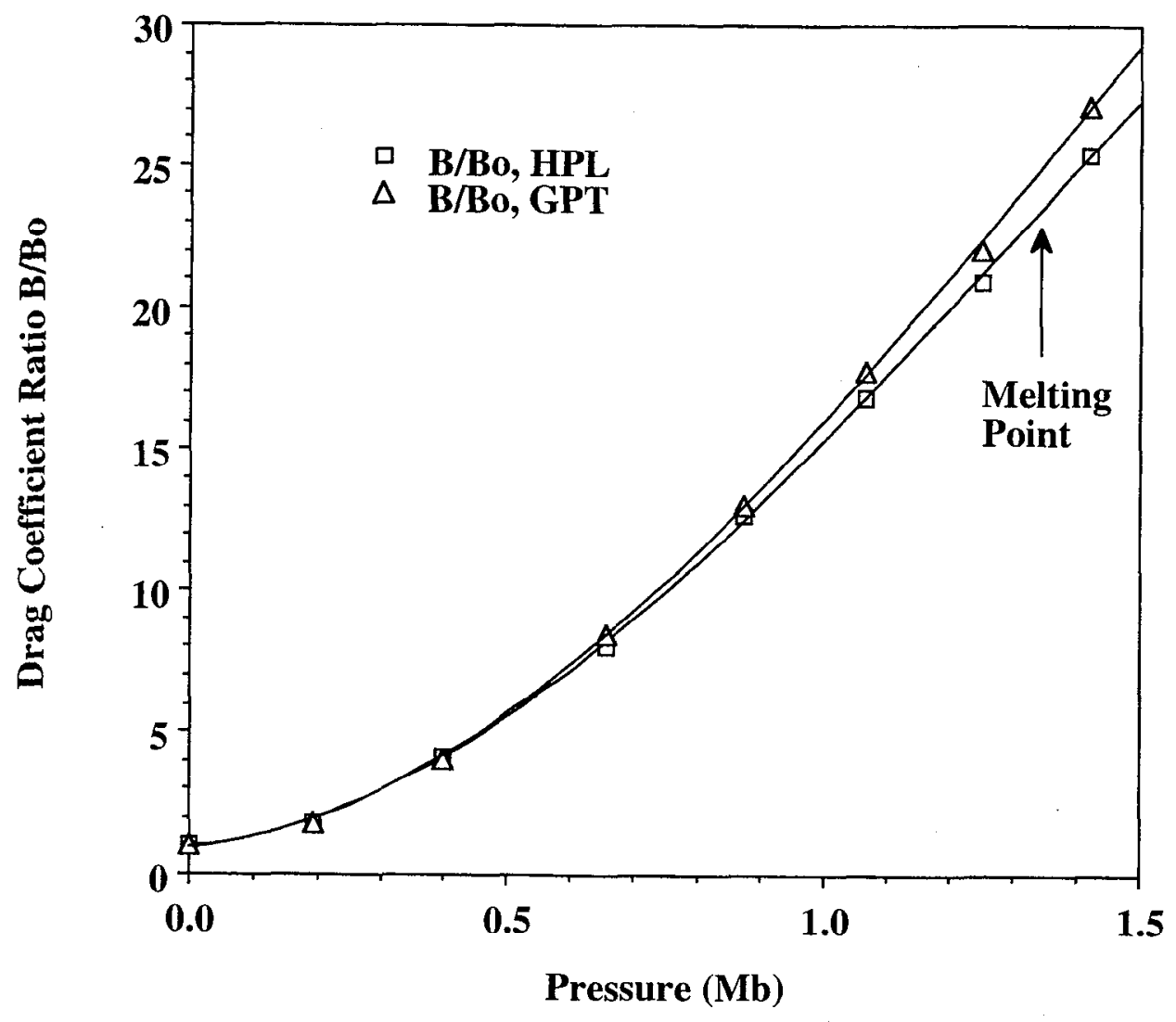

Figure 6. Variation of the Drag Coefficient with Pressure for Shock-Loaded Al. The Debye Temperature at standard conditions is $399 \mathrm{~K}$. 


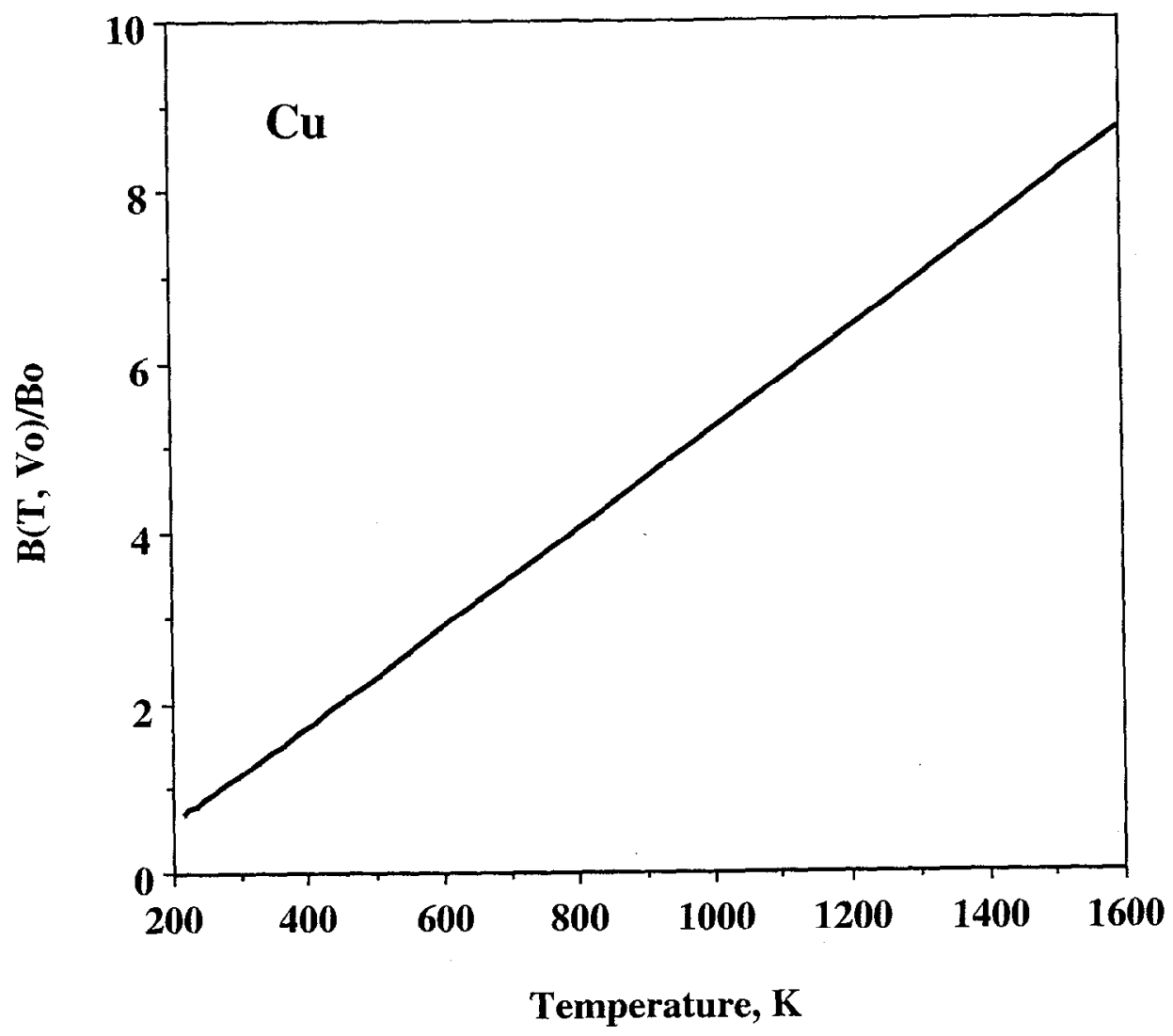

Figure 7. The temperature dependence of the phonon drag for $\mathrm{Cu}$ at constant volume. 


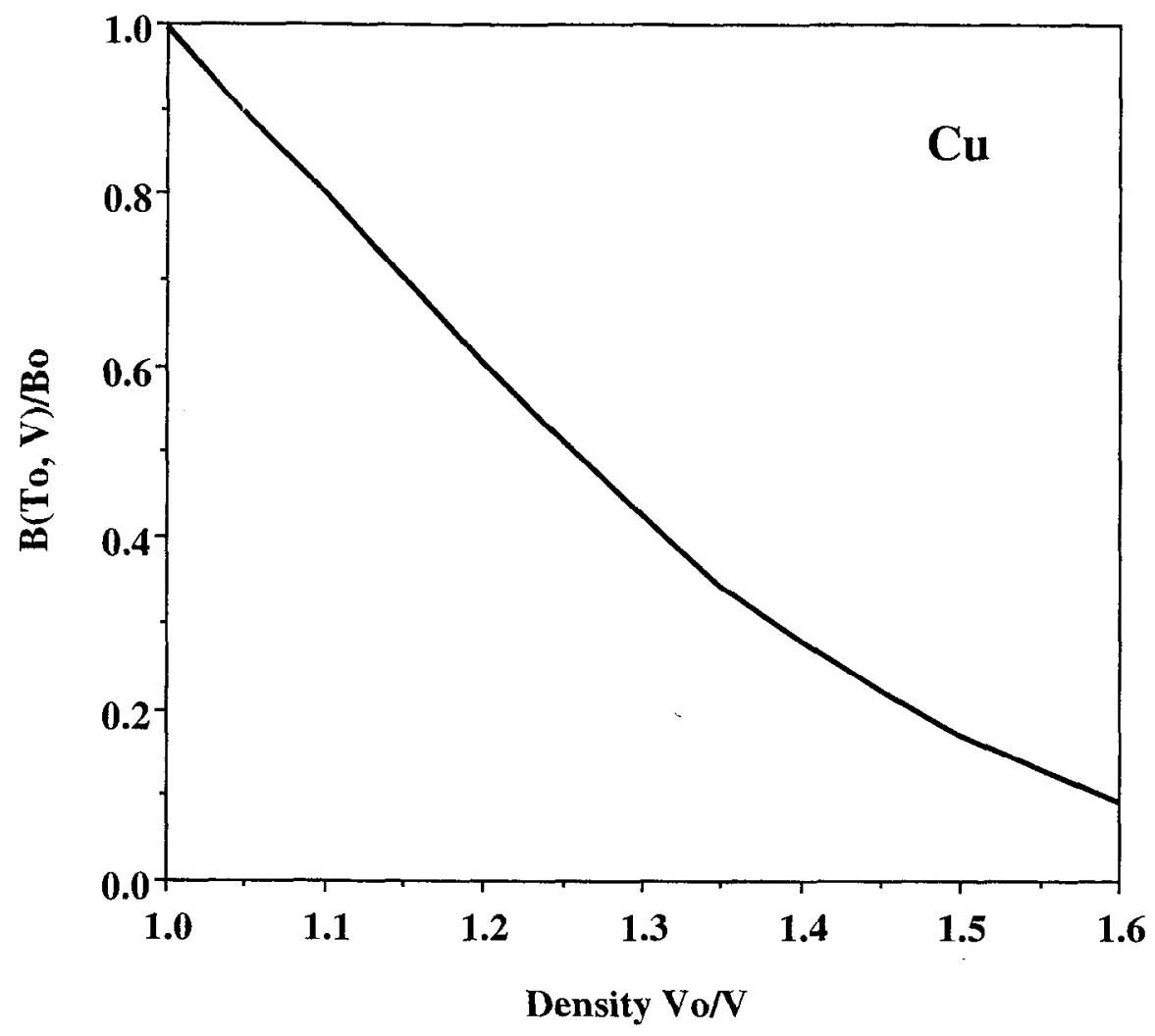

Figure 8. The volume dependence of the phonon drag for $\mathrm{Cu}$ at constant temperature. 


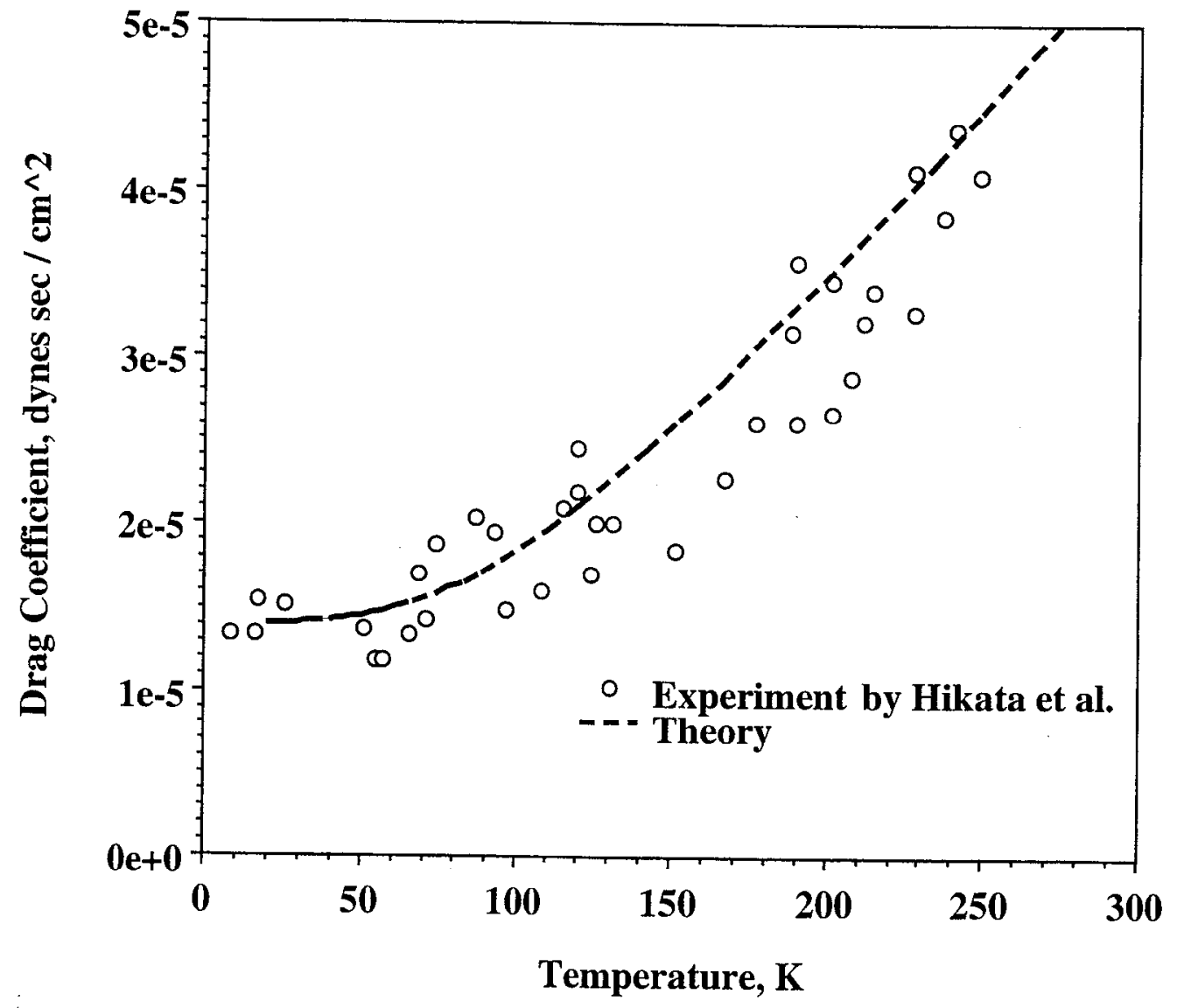

Figure 9. Comparison of experimental results with theoretical predictions. A constant electronic drag coefficient of $1.4 \mathrm{e}-5$ dynes sec/ $\mathrm{cm}^{2}$ and a scattering width of one Burgers vector is assumed. 